\title{
Brillouin scattering study of the ferroelectric phase transition in tris-sarcosine calcium chloride
}

\author{
Tomoyuki Hikita, ${ }^{*}$ Paul Schnackenberg, and V. Hugo Schmidt \\ Physics Department, Montana State University, Bozeman, Montana 59717
}

(Received 26 June 1984)

\begin{abstract}
Brillouin spectra from longitudinal phonons in ferroelectric tris-sarcosine calcium chloride propagating along [100], [010], and [001] have been measured as functions of temperature. Large anomalies were found in the Brillouin shift and linewidth in the [100] and [001] phonons. These anomalies are interpreted as arising from the linear coupling of the polarization and the phonons. From the temperature where the linewidth is maximum, the relaxation time of the polarization fluctuations is estimated to be $\tau=3.1 \times 10^{-12} /\left(T_{c}-T\right)$ sec, where $T_{c}$ is the ferroelectric transition temperature. We also observed anomalies in Brillouin shift and linewidth of the [010] phonons which propagate along the ferroelectric $b$ axis. These anomalies are interpreted as coming from electrostrictive coupling. The energy-relaxation time was estimated to be $\tau_{E}=2.5 \times 10^{-10} /\left(T-T_{c}\right) \mathrm{sec}$ in the paraelectric phase and $\tau_{E}=1.0 \times 10^{-9} /\left(T_{c}-T\right)$ sec in the ferroelectric phase, by comparing our Brillouin results with those of the ultrasonic measurements.
\end{abstract}

\section{INTRODUCTION}

Tris-sarcosine calcium chloride (TSCC), $\left(\mathrm{CH}_{3} \mathrm{NHCH}_{2} \mathrm{COOH}\right)_{3} \cdot \mathrm{CaCl}_{2}$, is a uniaxial ferroelectric, with a Curie temperature near $130 \mathrm{~K} .{ }^{1}$ At room temperature it has orthorhombic symmetry characterized by space group $D_{2 h}^{16}(P n m a)$ with four formula units per unit cell. ${ }^{2}$ In the ferroelectric (FE) phase this crystal remains orthorhombic with space group $C_{2 v}^{9}\left(\right.$ Pna $\left.2_{1}\right)$.

There are disputes concerning whether the FE transition of this crystal is order-disorder or displacive. Soft modes have been found in TSCC both in the paraelectric (PE) and FE phases. ${ }^{3-5}$ Scott and co-workers claim that this crystal is a textbook example of a displacive-type ferroelectric from their analysis of the results of millimeterwavelength spectroscopy. ${ }^{5}$ They could satisfactorily explain the temperature dependence and the dielectric constant in the above frequency region by a simple oscillator model. However, Chen and Schaack recently showed that their Raman and infrared spectra can be much better explained by the pseudospin-phonon-coupling theory. ${ }^{6,7}$ They concluded that TSCC and partially brominated TSCC (i.e., $\operatorname{TSCC}_{1-x} \mathbf{B}_{x}$ ) undergo phase transitions which are mostly of the order-disorder type. ${ }^{6,7}$ Furthermore, Schmidt showed that the pressure-temperature phase diagram $^{8}$ of TSCC can be interpreted by a cluster model, ${ }^{9}$ which indicates that this phase transition is partly of the order-disorder type. Since Brillouin scattering measures the velocity and attenuation of acoustic phonons in the $\mathrm{GHz}$ region, its use in TSCC helps to understand the dynamical behavior in this frequency region.

TSCC is the first ferroelectric for which the logarithmic correction has been unambiguously introduced in the analysis of the temperature dependence of the dielectric constant. $^{10}$ This could imply that the electric dipoledipole interaction is the main interaction which causes the ferroelectric phase transition. ${ }^{11}$ However, the spontaneous polarization is very small, ${ }^{1}$ whereas $T_{c}$ is quite large, so the ratio of dipolar to thermal energy (as calculated by Lajzerowicz and Legrand ${ }^{12}$ for some other ferroelectrics) is quite small, of order 0.01. According to Bruce, ${ }^{13}$ this implies that there should be logarithmic behavior only very near $T_{c}$, followed by a region of Ising critical behavior as temperature increases, and finally mean-field behavior will dominate at still higher temperature. Accordingly, the observation by Sandvold and Courtens ${ }^{10}$ of logarithmic behavior of dielectric susceptibility over a temperature range as wide as $50 \mathrm{~K}$ is surprising. Another strange characteristic of this crystal is that the TO and the LO phonons soften at the same temperature, ${ }^{5}$ which indicates that the short-range force and Coulomb force participate in the phase transition almost equally. ${ }^{4} \mathrm{Be}-$ cause of these contradictory indications of the strength of the dipole-dipole interaction, studies such as ours in which this interaction plays an important role are of particular interest.

Specifically, one could expect two interesting features in a Brillouin scattering study of TSCC. First, we can expect large anomalies in the velocity and attenuation of longitudinal phonons propagating along the directions perpendicular to the polar axis. These anomalies are caused by piezoelectric coupling in the FE phase. From these anomalies, we can evaluate the relaxation time of the polarization of the electric dipole system. ${ }^{14-16}$ Second, Sorge and Straube ${ }^{17}$ observed that at $20 \mathrm{MHz}$ the longitudinal ultrasonic wave propagation along the polar axis $b$ also shows large attenuation and velocity anomalies near $T_{c}$. In a system with electric dipole-dipole interactions, such anomalies are expected to occur not from piezoelectric coupling but from electrostrictive coupling. ${ }^{18,19}$ In this work we evaluate these relaxation times by investigating the anomalies in phonon velocity and attenuation from Brillouin scattering and comparing them with those from ultrasonic measurements.

\section{EXPERIMENTAL PROCEDURE}

Single crystals of TSCC were grown from an aqueous solution of pure sarcosine and reagent-grade $\mathrm{CaCl}_{2}$. Since this crystal has ferroelastic domains ${ }^{20}$ at room tempera- 
ture, single-domain portions were carefully examined with a polarizing microscope. Exceptionally clear parts were chosen from these crystals and two samples which have the form of a rectangular parallelepiped $3 \times 3 \times 2 \mathrm{~mm}^{3}$ were prepared. One sample was cut so that the longitudinal phonons $\vec{q} \|[100]$ and $\vec{q} \|[010]$ could be measured by right-angle scattering and the other was cut so that longitudinal phonons $\overrightarrow{\mathrm{q}} \|[001]$ and $\overrightarrow{\mathrm{q}} \|[010]$ could be measured. The two samples were polished and then masked together by a blackened copper foil with pinholes for the incident and the scattered light beams.

The masked sample pair was placed in a high-pressure optical cell described previously. ${ }^{21}$ The cell was filled with isopentane for index matching. The optical cell is surrounded by two radiation shields. The outer shield is directly connected to a liquid-nitrogen reservoir and the inner shield was temperature controlled at a temperature 6-10 K lower than that of the optical cell. Thus, the optical cell temperature could be controlled within $3 \mathrm{mK}$ during the measurement using a Lakeshore Cryotronics Model CSC-400 temperature controller.

A Lexel Model 95-2 argon-ion laser operating in a single mode at a wavelength of $514.5 \mathrm{~nm}$ and a power level of 200 to $300 \mathrm{~mW}$ was used as the light source. The scattered light was collected in a cone of $1^{\circ}$ and analyzed by a piezoelectrically scanned Burleigh Model 140 Fabry-Perot interferometer. Finesse optimization and drift control were achieved by a homemade control system using an AIM-65 microcomputer. The Fabry-Perot was scanned with a period of $10 \mathrm{sec}$ and the signal was stored in a multichannel analyzer. The signal accumulation times was 10 to $20 \mathrm{~min}$. Normally, the spacing of the étalon mirrors was adjusted to 40 to $50 \mathrm{~mm}$. The finesse of the spectrometer was typically 42 to 46 . Thus, the resolution of the spectrometer system was typically $77 \mathrm{MHz}$. The laser line broadening due to the jittering was claimed by the manufacturer to be about $10 \mathrm{MHz}$ [full width at half maximum (FWHM)].

For deducing the natural-phonon linewidth, the natural-phonon spectrum and the instrumental function were assumed to be Lorentzian distributions, and the broadening due to collection optics was assumed to have a rectangular distribution. In this case, the natural-phonon linewidth $\left(W_{\mathrm{ph}}\right)$ is given by ${ }^{22}$

$$
W_{\mathrm{ph}}=\left(W_{\text {obs }}^{2}-W_{\text {ang }}^{2}\right)^{1 / 2}-W_{\text {inst }},
$$

where $W_{\text {obs }}, W_{\text {inst }}$, and $W_{\text {ang }}$ designate the observed, instrumental, and collection optics linewidths, respectively.

\section{RESULTS AND DISCUSSION}

Figure 1 shows the temperature dependence of the Brillouin shift for longitudinal phonons propagating along [100], [010], and [001]. Each component shows an anomaly around $T_{c}$, with the [100] phonons showing the largest anomaly as well as the smallest Brillouin shift, and the [001] phonons showing the smallest anomaly. The shifts for the phonons with $\overrightarrow{\mathrm{q}} \|[100]$ and [010] barely show temperature dependence in the $\mathrm{PE}$ phase well above $T_{c}$, but the shift for $\vec{q} \|[001]$ phonons shows appreciable temperature dependence in the high-temperature region.

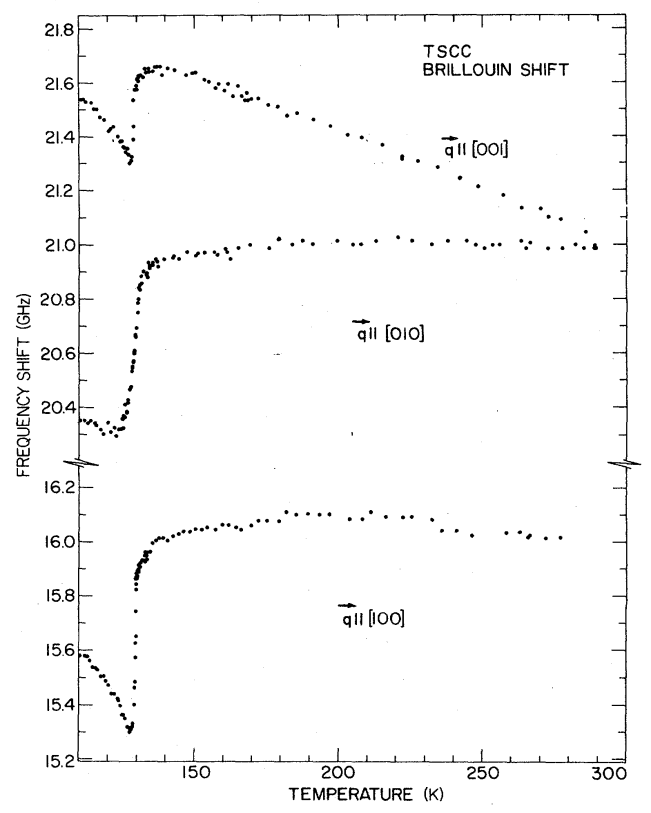

FIG. 1. Temperature dependence of Brillouin shifts of longitudinal phonons propagating along the $a, b$, and $c$ axes in TSCC. Scattering geometries are $x+y(z z)-x+y$, $x+y(z z) x-y$, and $y+z(x x) y-z$, respectively.

More detailed features near $T_{c}$ are shown in Figs. 2-4, where the temperature dependence of the Brillouin shift for the three phonon propagations is illustrated with an expanded temperature scale, together with the phonon decay rates. The temperature dependence of the Brillouin shift and the decay rate of the [100] phonons' is quite similar to that of the [001] phonons though the anomalies are smaller for the latter case. In the above two cases, the

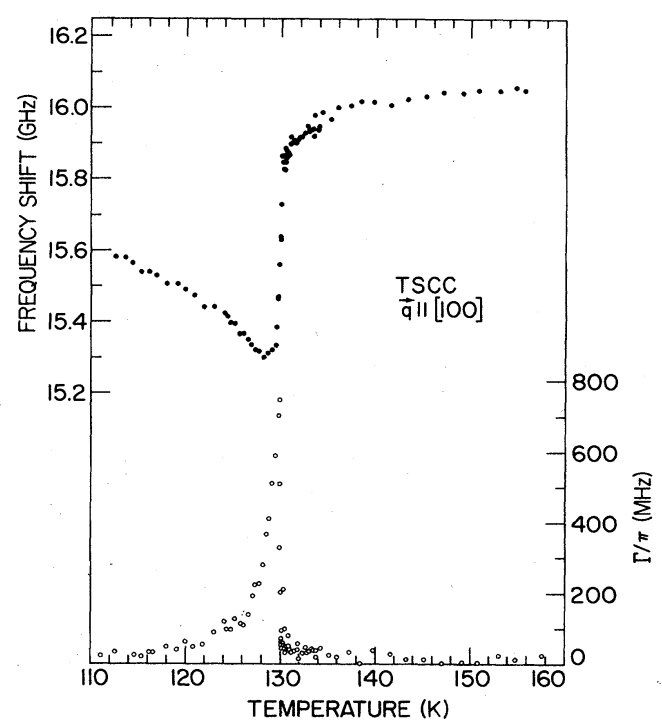

FIG. 2. Temperature dependence of Brillouin shift $(\bullet)$ and linewidth (O) (FWHM) of longitudinal phonons in TSCC propagating along the [100] direction. The scattering geometry is $x+y(z z)-x+y$. 


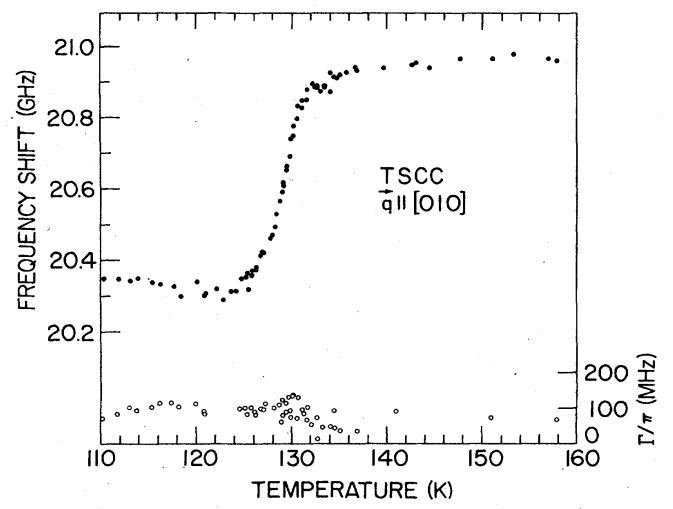

FIG. 3. Temperature dependence of Brillouin shift $(\bullet)$ and linewidth (O) (FWHM) of longitudinal phonons in TSCC propagating along the [010] direction. The scattering geometry is $x+y(z z) x-y$.

polarization and the strain couple bilinearly (LandauKhalatnikov type) in the FE phase. ${ }^{14}$ For these two phonon propagations, the velocity $v$ and the attenuation $\alpha$ in the FE phase are given by ${ }^{15,16,23}$

$$
\begin{aligned}
& v^{2}=v_{\infty}^{2}-\left[\left(v_{\infty}^{2}-v_{0}^{2}\right) /\left(1+\omega^{2} \tau_{0}^{2} t^{-2}\right)\right], \\
& \alpha=\left[\left(v_{\infty}^{2}-v_{0}^{2}\right) / 2 v^{3}\right]\left[\omega^{2} \tau_{0} t^{-1} /\left(1+\omega^{2} \tau_{0}^{2} t^{-2}\right)\right],
\end{aligned}
$$

where $t$ is the reduced temperature $\left(T_{c}-T\right) / T_{c}$, and $\tau_{0}$ is the individual-dipole relaxation time in the expression $\tau=\tau_{0} t^{-1}$ for the relaxation time $\tau$. The velocities $v_{\infty}$ and $v_{0}$ designate the high- and low-frequency limit velocities, respectively. The Brillouin shift $(\Delta v)$ is given by

$$
\Delta v=(v v / c)\left(n_{i}^{2}+n_{s}^{2}\right)^{1 / 2},
$$

where $v$ is the incident light frequency, $c$ is the speed of light in vacuum, and $n_{i}$ and $n_{s}$ denote refractive indices for the incident and the scattered light, respectively. The relaxation between the phonon decay rate $\Gamma$ or the Brillouin linewidth (FWHM) $\Gamma / \pi$ and the attenuation coefficient $\alpha$ is given by ${ }^{22}$

$$
\Gamma / \pi=\alpha v / \pi \text {. }
$$

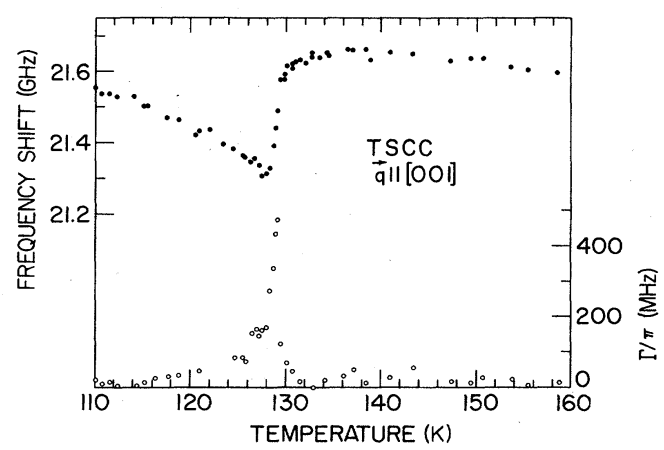

FIG. 4. Temperature dependence of Brillouin shift $(\bullet)$ and linewidth (O) (FWHM) of longitudinal phonons in TSCC propagating along the [001] direction. The scattering geometry is $y+z(x x) y-z$.

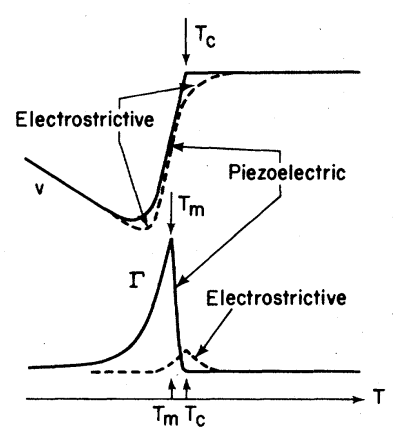

FIG. 5. Anomalies in the velocity and the attenuation caused by piezoelectric coupling (Landau-Khalatnikov type) and by electrostrictive coupling (fluctuations) for longitudinal phonons propagating perpendicular to the polarization axis.

From Eq. (2) we notice that the phase transition is marked by a quick drop in the velocity with decreasing temperature. However, experimentally the [100] and [001] phonon velocities do not exhibit such sharp drops at $T_{c}$, but rather show slow rounding. These anomalies are attributed to the quadratic coupling of the polarization and the strain or in other words, electrostrictive coupling ${ }^{14,19}$ (fluctuation damping). Such contributions to the anomalies of the velocity and the attenuation are schematically illustrated in Fig. 5. As we see from Fig. 5, the temperature $T_{m}$ at which the attenuation coefficient is maximum does not equal $T_{c}$, but occurs somewhat below $T_{c}$. From Eqs. (2) and (3) we obtain the relation

$$
\left(T_{c}-T_{m}\right) / T_{c}=\omega \tau_{0} \text {. }
$$

By this relation we can calculate the elementary relaxation time $\tau_{0}$. Although the clear indication of $T_{c}$ is blurred by the additional small anomaly from the electrostrictive contribution, we can use the steepest dropping point of the velocity as $T_{c}$. From the velocity and attenuation for the above two cases, we obtain the following results: For the $\overrightarrow{\mathrm{q}}||[100]$ phonons,

$$
T_{c}-T_{m}=0.3 \mathrm{~K}, \tau_{0}=2.4 \times 10^{-14} \mathrm{sec}
$$

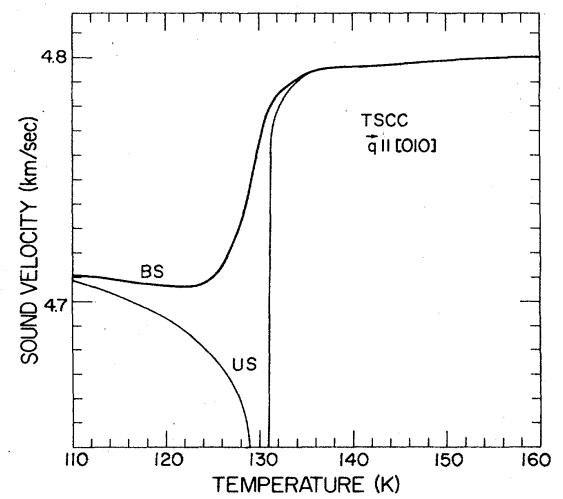

FIG. 6. Temperature dependence of sound velocity in TSCC for Brillouin scattering (BS) at $16 \mathrm{GHz}$ and for ultrasonic (US) measurements at $20 \mathrm{MHz}$. The velocity scale for the Brillouin scattering was adjusted to that of the ultrasonic measurements. 


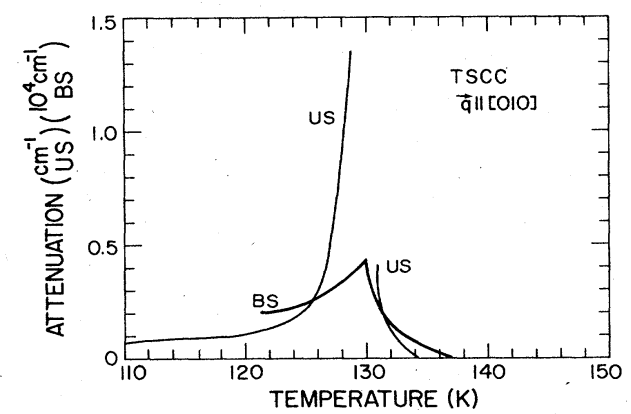

FIG. 7. Temperature dependence of attenuation in TSCC for Brillouin scattering (BS) at $16 \mathrm{GHz}$ and for ultrasonic (US) measurements at $20 \mathrm{MHz}$.

and for the $\overrightarrow{\mathrm{q}} \|[001]$ phonons,

$$
T_{c}-T_{m}=0.4 \mathrm{~K}, \tau_{0}=2.3 \times 10^{-14} \mathrm{sec} .
$$

The above two results agree well. We will use their average, $\tau_{0}=2.4 \times 10^{-14} \mathrm{sec}$, as the elementary relaxation time of the polarization fluctuations in TSCC.

The relaxation time of the polarization fluctuations obtained from the $\vec{q} \|[100]$ and $\vec{q} \|[001]$ phonons is short compared to that of other order-disorder ferroelectrics. For instance, $\tau_{0}$ is $1.2 \times 10^{-13} \mathrm{sec}$ for potassium dihydrogen phosphate (KDP), ${ }^{24} 1.3 \times 10^{-12}$ sec for deuterated KDP (DKDP), ${ }^{24}$ and $9.6 \times 10^{-14} \mathrm{sec}$ for triglycine sulfate (TGS). ${ }^{15}$

Next we consider the temperature dependence of the Brillouin shift and the phonon decay rate for the longitudinal phonons parallel to the direction of the spontaneous polarization [010]. In this case, polarization fluctuations do not couple bilinearly to the strain, because of the appearance of the depolarization field in the longitudinal polarization wave. ${ }^{18,19}$ Therefore, the anomalies in the velocity and attenuation of these phonons should be explained by quadratic coupling of the polarization to the strain (electrostriction) in both PE and FE phases.

We now compare the velocity and attenuation of the acoustic waves in the $\mathrm{GHz}$ and $\mathrm{MHz}$ regions. Ultrasonic velocity and attenuation measurements have been performed on this crystal by Sorge and Straube. ${ }^{17}$ Their results are reproduced in Figs. 6 and 7, together with our Brillouin results which are converted to velocity and attenuation scales.

We use the velocities obtained by Brillouin scattering and ultrasonic measurements for $v_{\infty}$ and $v_{0}$, respectively. We assume the simplest form for the relaxation time to be $\tau_{E}=\tau_{0}^{E}\left|t^{-1}\right|$, where $\tau_{0}^{E}$ is the elementary relaxation time. The $E$ designates that these relaxation times are related to energy rather than polarization fluctuations. By putting into Eq. (3) the values of the attenuation coefficient of the ultrasonic measurements at the temperatures $T-T_{c}$ $=+0.9$ and $-1.3 \mathrm{~K}(2.7$ and $11.1 \mathrm{~dB} / \mathrm{cm}$, respectively $)$, we obtain the following values of the relaxation time: and

$$
\tau_{0}^{E}=1.9 \times 10^{-12} \mathrm{sec} \text { for } T>T_{c}
$$

$$
\tau_{0}^{E}=7.7 \times 10^{-12} \mathrm{sec} \text { for } T<T_{c} .
$$

The elementary relaxation times for this case are 2 orders of magnitude larger than those for the polarization relaxation. This is the reason why we cannot observe marked broadening in the Brillouin linewidth for the [010] phonons. Using the above $\tau_{0}^{E}$ values we estimate the Brillouin linewidth in attenuation units to be $280 \mathrm{~cm}^{-1}$ at $T-T_{c}=0.9 \mathrm{~K}$ and $67 \mathrm{~cm}^{-1}$ at $T_{c}-T=1.3 \mathrm{~K}$. These values are too small when compared to the observed linewidths. For $\vec{q} \|[010]$ we find that the relaxation time is so long, $\omega \tau \gg>1$, that the relations (2) and (3) are no longer valid. ${ }^{22}$ In this high-frequency regime we need a more developed mode-mode coupling theory to calculate the relaxation time. ${ }^{25}$ However, in this paper we shall only give a rough estimate of the relaxation time. In the case of $\vec{q} \|[010]$ propagation the coupling of the order parameter and the strain is quadratic, so the anomaly in the sound propagation is considered to arise from an origin similar to that of the specific heat. ${ }^{26}$ If we want to study the relaxation time in more detail, we have to measure the attenuation and the velocity at various frequencies. This could be achieved by performing Brillouin scattering at low frequencies ${ }^{22}$ (small-angle scattering).

Chen and Schaack ${ }^{6,7}$ obtained $\tau_{0}=2.7 \times 10^{-13} \mathrm{sec}$ from their analysis of the pseudospin-phonon coupling of Raman and infrared spectra. This value is 1 order of magnitude larger than ours. Deguchi et al. ${ }^{27}$ showed that the relaxation time in TSCC is expressed by $\tau=3.8 \times 10^{-13}$ $\left(\epsilon_{s}-\epsilon_{\infty}\right)$ sec from their dielectric dispersion measurements. If we use the values $C^{+}=30$ to $50 \mathrm{~K}$ for the Curie constant ${ }^{21,28}$ and the relation $C^{+} / C^{-}=2$, we obtain the values $\tau_{0}=4.4$ to $8.5 \times 10^{-14} \mathrm{sec}$ for the elementary relaxation time which is in relatively good agreement with our value. Though we can obtain an orderparameter relaxation time from the Brillouin scattering experiment, this does not necessarily mean that TSCC undergoes an order-disorder phase transition. It is also possible to adopt the interpretation that the soft-phononmode frequency $\omega_{0}$ decreases and the damping constant $\gamma$ diverges as $T_{c}$ is approached. In such an extremely damped case, the system has the same correlation function as that of the relaxational mode where the effective relaxation time is given by $\gamma / \omega_{0}^{2}$.

The most interesting feature of the Brillouin scattering in TSCC is the anomaly in the velocity and the decay rate of the [010] phonons. We estimated roughly the energy relaxation time in both the PE and FE phases. For convenience, we assumed that the critical index for $\tau_{E}$ is the same as that of the polarization relaxation time. General$1 \mathrm{y}$, this is not true. It would be worthwhile to obtain the precise critical index for this relaxation time. For this purpose, low-frequency Brillouin scattering is recommended also. This crystal is claimed to exhibit tricritical behavior $^{29}$ at high hydrostatic pressure. Investigation of the change in the critical index of the relaxation time around such a multicritical point is very interesting and is now planned in our laboratory.

\section{ACKNOWLEDGMENTS}

We thank Professor J. F. Scott for a helpful discussion. This work was supported in part by National Science Foundation Grant No. DMR-8205280. 
*On leave from Tohoku University, Sendai 980, Japan.

${ }^{1}$ Y. Makita, J. Phys. Soc. Jpn. 20, 2073 (1965).

${ }^{2}$ T. Ashida, S. Bando, and M. Kakudo, Acta Crystallogr. Sect. B 28, 1560 (1972).

${ }^{3}$ S. D. Prokhorova, G. A. Smolensky, I. G. Siny, E. G. Kuzminov, V. D. Mikvabia, and H. Arndt, Ferroelectrics 25, 629 (1980).

${ }^{4}$ G. E. Feldkamp, J. F. Scott, and W. Windsch, Ferroelectrics 39, 1163 (1981).

${ }^{5}$ G. V. Kozlov, A. A. Volkov, J. F. Scott, G. E. Feldkamp, and J. Petzelt, Phys. Rev. B 28, 255 (1983).

${ }^{6}$ T. Chen and G. Schaack, J. Phys. C 17, 3801 (1984).

${ }^{7}$ T. Chen and G. Schaack, J. Phys. C 17, 3821 (1984).

${ }^{8}$ V. H. Schmidt, Solid State Commun. 35, 649 (1980).

${ }^{9}$ V. H. Schmidt, Ferroelectrics 39, 1151 (1981).

${ }^{10}$ E. Sandvold and E. Courtens, Phys. Rev. B 27, 5660 (1983).

${ }^{11}$ A. I. Larkin and D. E. Khmel'nitskii, Zh. Eksp. Teor. Fiz. 56, 2087 (1969) [Sov. Phys.-JETP 29, 1123 (1969)].

12J. Lajzerowicz and J. F. Legrand, Phys. Rev. B 17, 1438 (1978).

${ }^{13}$ A. D. Bruce, Adv. Phys. 29, 111 (1980).

${ }^{14}$ W. Rehwald, Adv. Phys. 22, 721 (1973).

${ }^{15}$ R. W. Gammon and H. Z. Cummins, Phys. Rev. Lett. 17, 193 (1966).

${ }^{16}$ T. Yagi, M. Tokunaga, and I. Tatsuzaki, J. Phys. Soc. Jpn. 40, 1659 (1976).
${ }^{17} \mathrm{G}$. Sorge and U. Straube, Ferroelectrics 21, 533 (1978).

${ }_{18}$ M. A. Krivoglaz, Fiz. Tverd. Tela (Leningrad) 5, 3439 (1963) [Sov. Phys.-Solid State 5, 2526 (1964)].

${ }^{19}$ K. A. Minaeva, B. A. Strukov, and K. Varnstorff, Fiz. Tverd. Tela (Leningrad) 10, 2125 (1968) [Sov. Phys.-Solid State 10, 1665 (1969)].

${ }^{20}$ A. Sawada, Y. Makita, and Y. Takagi, J. Phys. Soc. Jpn. 42, 1918 (1977).

${ }^{21}$ M. Yamada and V. H. Schmidt, Rev. Sci. Instrum. 49, 1226 (1978).

${ }^{22}$ T. Hikita, J. Phys. Soc. Jpn. 53, 1513 (1984).

${ }^{23}$ K. F. Herzfeld and T. A. Litovitz, Absorption and Dispersion of Ultrasonic Waves (Academic, New York, 1959).

${ }^{24}$ I. Tatsuzaki, M. Kasahara, M. Tokunaga, and H. Tanaka, Ferroelectrics 39, 1049 (1981).

${ }^{25} \mathrm{~K}$. Kawasaki, in Phase Transitions and Critical Phenomena, edited by C. Domb and M. S. Green (Academic, New York, 1979), Vol. 5A, Chap. 4.

${ }^{26}$ A. Lopez-Echarri and M. J. Tella, J. Phys. D 14, 71 (1981).

${ }^{27}$ K. Deguchi, N. Aramaki, E. Nakamura, and K. Tanaka, J. Phys. Soc. Jpn. 52, 1897 (1983).

${ }^{28}$ U. Straube, G. Sorge, and N. R. Ivanov, Phys. Status Solidi A 73, 467 (1982).

${ }^{29}$ H. Reichelt, W. Windsch, and A. Sienkiewicz, Ferroelectrics 34, 195 (1981). 